\title{
PROTEOLYTIC CLEAVAGE OF THE MURINE CORONAVIRUS SURFACE GLYCOPROTEIN IS NOT REQUIRED FOR ITS FUSION ACTIVITY
}

\author{
Roland Stauber, Michael Pfleiderer and Stuart Siddell \\ Institut für Virologie \\ Universität Würzburg \\ Versbacherstr.7 \\ 8700 Würzburg
}

\section{SUMMARY}

The surface glycoprotein (S) of the murine hepatitis coronavirus MHV normally undergoes proteolytic cleavage during transport to the cell surface. To determine whether the cleavage of the MHV-JHM S glycoprotein is required to activate its ability to fuse cellular membranes, the protease recognition sequence in a cDNA copy of the $S$ gene was altered from Arg-Arg-Ala-Arg-Arg into Ser-Val-Ser-Gly-Gly by site directed mutagenesis. The mutated and wild type $S$ genes were expressed by means of recombinant vaccinia viruses and it could be shown that the mutated $S$ protein was not cleaved when it was expressed in mouse DBT cells, in contrast to the wild type S protein. Nevertheless, the non-cleaved $S$ protein induced extensive syncytium formation in mouse DBT cells. These results clearly indicate that the non-cleaved form of the MHV S protein is able to mediate cell membrane fusion. Thus, proteolytic cleavage is not an absolute requirement for its fusion function.

\section{INTRODUCTION}

The coronaviruses are a group of pleomorphic, enveloped, positive stranded RNA viruses associated with diseases of economic importance in both animals and humans (1). The murine coronavirus (murine hepatitis virus [MHV]) has a genomic RNA of 32 $\mathrm{kb}(2)$ which encodes four major structural proteins: the nucleocapsid protein $\mathrm{N}$, the membrane glycoprotein $M$, the hemagglutinin-esterase $\mathrm{HE}$, and the surface or spike glycoprotein S (3). As the name suggests, the MHV S protein forms the projecting spikes or peplomers on the surface of the virus particle. The $S$ protein is synthesized as a cotranslationally glycosylated precursor which then undergoes oligomerization and carbohydrate processing. The polypeptide is also proteolytically cleaved by a host cell enzyme which recognizes a basic sequence, Arg-Arg-Ala-Arg-Arg, located approximately in the middle of the molecule (4). Thus, the mature spike structure consists of two $S$ protein molecules, each of which is a heterodimer comprised of two non-covalently bound subunits. The $S$ protein is able to mediate attachment of the virus to the cell surface and the fusion of cellular and viral membranes (5).

The entry of the enveloped virus particle is thought to be initiated via an interaction between a virus glycoprotein and the cellular receptor, which is followed by fusion of the viral envelope with the host cell plasma membrane or a host endosomal membrane. A common characteristic of fusion proteins is cleavage, which is thought to expose an internal hydrohobic domain that can interact with the lipid bilayer. With the exception of 
rhabdoviruses, the majority of viral fusion proteins must be cleaved to be functional. This has been demonstrated for the influenza hemagglutinin (6) and human immunodeficiency virus type 1 (HIV-1) gp 160 (7). The lines of evidence which argue that also for the MHV-S protein cleavage is required for the fusion activity are as follows. First, the addition of exogenous protease to the growth medium can enhance plaque and syncytium formation by some strains of MHV (8). Second, MHV virions grown in 17Cl-1 cells contain approximately equal amounts of cleaved and uncleaved S protein and are unable to mediate fusion from without. After treatment with trypsin in vitro (which converts the majority of the $S$ protein to the cleaved form), they acquire this ability (9). Third, the addition of protease inhibitors to the medium of MHV-infected cells causes a delay in the onset of cell fusion (10). The fact however that a number of coronavirus $S$ proteins do not undergo post-translational cleavage and are nevertheless able to initiate infection and induce syncytium formation (11) prompted us to reinvestigate the relationship of MHV S protein cleavage to fusion. The experiments reported here lead us to the conclusion that proteolytic cleavage is not an absolute requirement for fusion activity.

\section{MATERIAL AND METHODS}

\section{Cells and viruses}

HeLa cells, DBT cells, Sac(-) cells and TK" 143B cells were grown in monolayers in minimal essential medium containing $10 \%$ heat-inactivated foetal calf serum, glutamine, antibiotics and non-essential amino acids. The MHV used in this study is the MHV Wb1 isolate described in Schwarz et al. (12). Vaccinia virus (WR strain) and the recombinant vaccinia virus, vTF7-3 (13) were plaque purified twice and grown to stocks of approximately $2 \times 10^{9}$ p.f.u./ml in HeLa cells as described by Mackett et al. (14).

\section{cDNA cloning and sequence analysis}

Poly(A)-containing RNA was isolated from MHV infected Sac(-) cells and cDNA synthesis was essentially done by the method of Gubler \& Hoffman (15) using MHVspecific oligonucleotides. Recombinant clones were identified by colony hybridisation with two MHV-S specific oligonucleotides. The cDNA of one clone, $\mathrm{pBS}+/ \mathrm{S}$, which gave a positive signal with both oligonucleotides in the colony hybridization was sequenced. The cDNA insert of $\mathrm{pBS}+/ \mathrm{S}$ was cloned into the vaccinia transfer vector, pTF7-5 (16). The correct clone, pTF7-5/S, was identified by colony hybridization, restriction endonuclease digestion and sequence analysis.

\section{Oligonucleotide-directed mutagenesis}

Mutagenesis was performed using the Amersham system which is based on the method of Nakamaye. Briefly, the cDNA insert of $\mathrm{pBS}+/ \mathrm{S}$ was cloned into the M13mp19 RF DNA which had been cut with the same enzymes. The resulting construct M13mp19/S, was used to transform competent E. coli TG-1 cells and single-strand DNA was isolated. Site directed mutagenesis was performed on the single stranded M13mp19/S-DNA according to the manufacturer.

\section{Cloning of the mutated MHV-S gene}

After performing the oligonucleotide-directed mutagenesis a 632 bp DNA fragment containing the mutated cleavage site, was cut out of double stranded M13mp19/S-Mut. DNA and used to replace the wild type cleavage site sequence in the $\mathrm{pBS}+/ \mathrm{S}$ DNA. The resulting $\mathrm{pBS}+/ \mathrm{S}-\mathrm{Mut}$. DNA was cloned into the vaccinia transfer vector pTF7.5 (16).

\section{Isolation of vaccinia virus recombinants}

Recombinant vaccinia virus were constructed by established procedures. Briefly, HeLa cells were infected at a low multiplicity of infection with wild type vaccinia virus strain WR and transfected with different recombinant transfer plasmids by using the 
calcium phosphate method. Progeny virus was plaqued on human TK--cells in the presence of 5-bromodeoxyuridine $(25 \mu \mathrm{g} / \mathrm{ml})$. Recombinant vaccinia viruses were identified by DNA hybridization using specific probes and indirect immunofluorescence. Plaque purification and screening were repeated three times before stocks of recombinant viruses were grown in HeLa cells.

\section{SDS-PAGE and Immunoblotting}

SDS-PAGE was done under reducing conditions on $7.5 \%$ polyacrylamide gels according to the method of Laemmli. Western immunoblots were stained with undiluted hybridoma tissue culture supernatant from the MHV-S protein specific hybridoma $11 \mathrm{~F}$ (17), followed by peroxidase-linked rabbit anti-mouse immunoglobulin and the substrate 4-chloronaphthol.

\section{Indirect immunofluorescence}

DBT cell monolayers on glass coverslips were infected with recombinant vaccinia viruses at an m.o.i. of 1 p.f.u./cell. After $2 \mathrm{~h}$, the virus containing medium was replaced with medium or medium containing $0.5 \%$ ascites fluid. The ascites fluid contained monoclonal antibody $11 \mathrm{~F}$ (17) or a monoclonal antibody specific for the MHV 5b protein. At $14 \mathrm{~h}$ post-infection, the cells were washed twice with ice-cold PBS, fixed with acetone-methanol (1:1), washed again with ice-cold PBS and incubated for $16 \mathrm{~h}$ at $4^{\circ} \mathrm{C}$ with tissue culture supernatant containing a mixture of 12 monoclonal antibodies which recognize linear or discontinuous determinants on the MHV S protein (18). The cells were then washed extensively at room temperature with PBS and incubated for $2 \mathrm{~h}$ with fluorescein-conjugated goat anti-mouse immunoglobulin $(0.5 \%$ in PBS containing 5\% goat serum). After extensive washing with PBS, the coverslips were mounted in $90 \%$ glycerol ( $\mathrm{pH} \mathrm{8.0)}$ ) and fluorescence micrographs were taken on a Leitz Aristoplan microscope.

\section{RESULTS}

The cDNA insert of $\mathrm{pBS}+/ \mathrm{S}$ was sequenced and found to extend from a position 29 nucleotides downstream of the $S$ protein gene, to a position 63 nucleotides upstream of the initiation codon. The sequence upstream of the initiation codon corresponds to the MHV leader sequence showing that the cDNA was copied from mRNA 3 (4). In order to demonstrate the fusogenic activity of the $S$ protein encoded by this cDNA, we performed a transient expression assay using a system based on a recombinant vaccinia virus that synthesizes T7 RNA polymerase (16). DBT cells which have been infected with vTF7-3 and transfected with pTF7.5/S DNA show extensive syncytium formation. In contrast, cells which have been infected with vTF7-3 and transfected with pTF7-5 DNA show no syncytium formation. This result demonstrates that the $S$ protein gene used in these studies is functional and is able to induce fusion from within in the absence of other viral proteins.

Having confirmed that the recombinant MHV-JHM S protein possesses fusion activity we addressed the question if cleavage of the $S$ protein is necessary to cause syncytia formation. For this purpose we changed by means of site directed mutagenesis the basic recognition sequence Arg-Arg-Ala-Arg-Arg (amino acids 624-628), required for the cleavage by a host cell protease, into Ser-Val-Ser-Gly-Gly. In the resulting plasmid pTF7.5/S-Mut. the changed sequence was confirmed by sequence analysis and the DNA used for the generation of recombinant Vaccinia virus.

Using the Vaccinia virus system it was possible to monitor the expression of the wild type and the mutant MHV-JHM S gene. For this purpose lysates of DBT cells infected with the recombinant vaccinia virus vTF7-3 and the vT7.5/S or vTF7.5/S-Mut. at a m.o.i. of 10 respectively were analyzed on $7.5 \%$ polyacrylamide gels and immunoblotting was performed. In Fig. 1 the uncleaved and the cleaved form of the $S$ protein can be seen for the expressed wild type MHV-JHM S gene (lane 3), whereas no cleavage products are visible for the mutant $S$ protein (lane 2). 


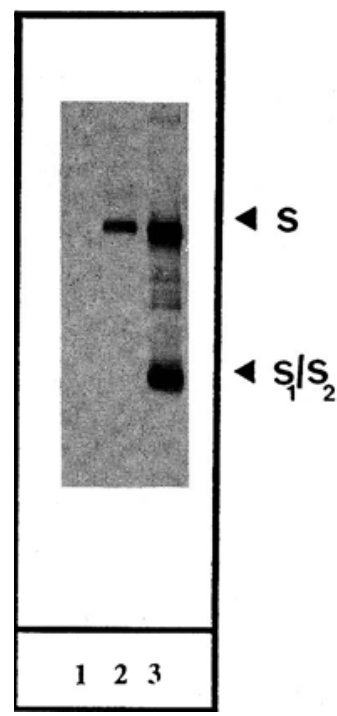

Figure 1 Western blot analysis of the wild type and mutated MHV S protein expressed by means of recombinant vaccinia virus. Lane 1 vTF7-3 infected, lane 2 vTF7-3 and vTF7-5/SMut. infected, lane 3 vTF7-3 and vTF7-5/S infected.

Cleavage of viral fusion glycoproteins is known to be prerequisite to activate their biological function. To determine wether this is also the case for the MHV-JHM S glycoprotein, we tested the wild type and the mutated S protein for their ability to cause syncytia formation.

In Fig. 2 DBT cells which had been infected with vTF7-3 as well as vTF7-5/S or vTF7-5/S-Mut were examined by indirect immunofluorescence $14 \mathrm{~h}$ post infection. Figure 2 (panel A, B) demonstrates that the wild type and the mutated $S$ protein are able to elicit syncytia formation in DBT cells to the same extent.

The fact that an anti S MoAb, which inhibits MHV-JHM mediated fusion, is able to block the fusion activity of the wild type as well as the mutant S protein (panel C, D), proves that this cell-to-cell fusion is specifically caused by the expression of the recombinant $S$ proteins. As expected the control MoAb against the MHV $5 b$ has no inhibitory effect on the fusion activity (panel E, F).

\section{DISCUSSION}

Although virus mediated cell-fusion has been studied quite extensively, little is known about the mechanistic details of the initial interaction between the viral fusion protein and the lipid bilayer. In many enveloped virus families cleavage of the fusion glycoprotein is essential for its function. Besides the cleavage event another important factor is the $\mathrm{pH}$ dependence of fusion, which often reflects the route of virus entry. The alphaviruses and the orthomyxoviruses bind to plasma membrane receptors and are internalized via the endocytic pathway which has an acidic $\mathrm{pH}$. The low $\mathrm{pH}$ causes conformational changes in the fusion glycoprotein and triggers fusion of the viral envelope with the endosomal membrane to release the viral RNA into the cytoplasm. On the other hand viruses, which enter the cell via direct fusion between the cellular plasma membrane and the viral membrane such as retroviruses or coronaviruses (19), possess fusion glycoproteins which are functional at neutral $\mathrm{pH}$. To render the situation even more complex physical parameters such as the lipid composition of the host cell membrane seems to play a role in virus induced fusion (20). 


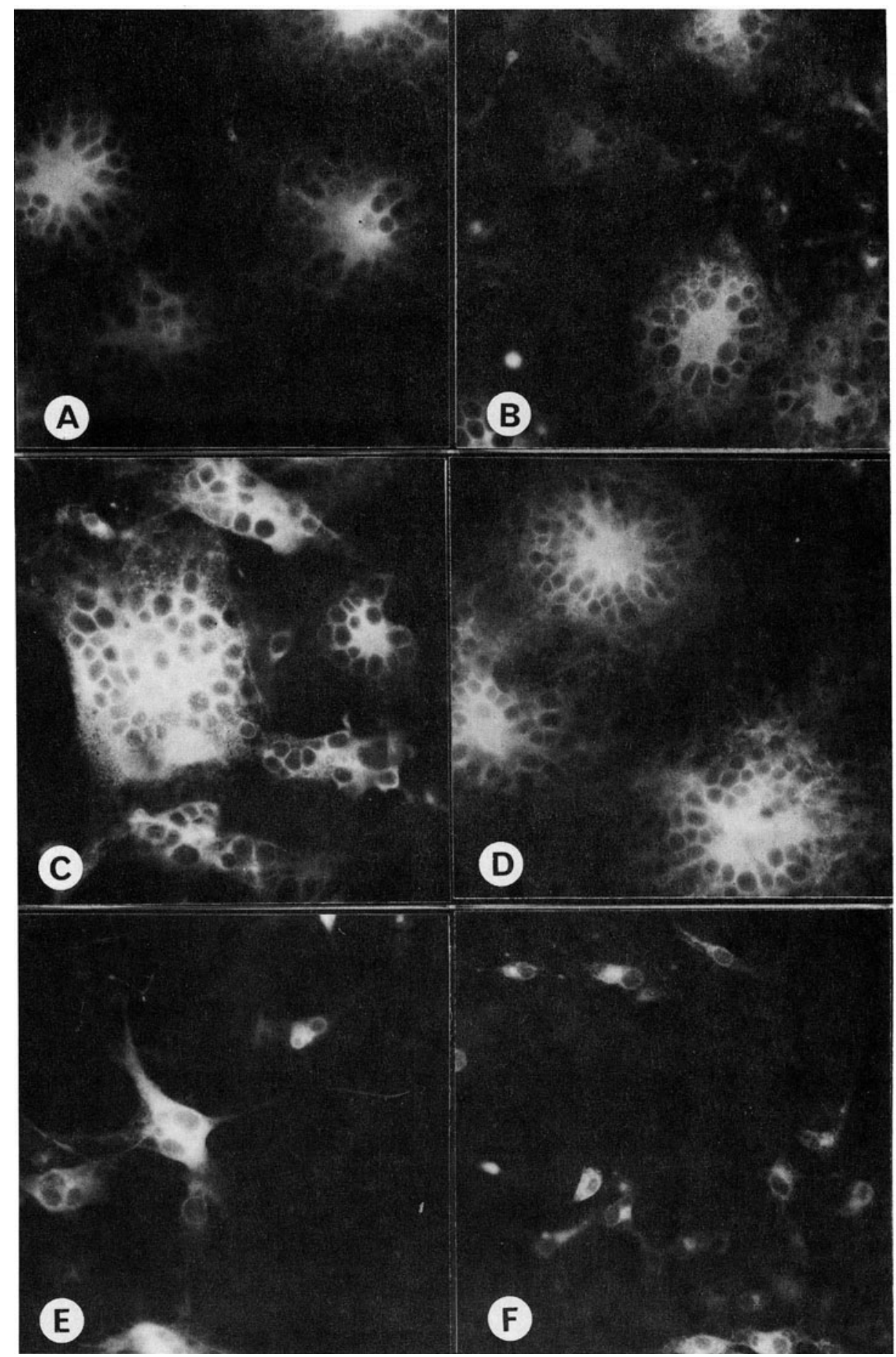

Figure 2 Fusion activity of the wild type and mutated $S$ proteins.

Panel A vTF7-5/S infected, medium; panel B vTF7-5/S-Mut., medium

Panel C vTF7-5/S infected, medium with Mab 5b; panel D vTF7-5/S-Mut, medium with Mab 5b

Panel E vTF7-5/S infected, medium with Mab 11F; panel F vTF7-5/S-Mut., medium with Mab $11 \mathrm{~F}$ 
The results reported in this paper show that the putative cleavage site Arg-Arg-AlaArg-Arg is the only site which is used because mutation of this sequence abolishes cleavage. Therefore no other potential protease recognition sites, e.g. the recognition site for thermolysin which was suggested by Frana, et al. (10) play a role in the cleavage of the MHV-JHM S protein in DBT cells.

Finally it could be demonstrated that the MHV-JHM S protein does not need to be cleaved to activate its fusion function. Although we conclude that proteolytic cleavage of the MHV S protein is not required for its fusion activity, we do not wish to imply that this processing event is without significance. The cleavage site motif is well conserved amongst MHV strains (4) and processing of the $S$ protein precursor occurs in a wide variety of murine cell lines. One possibility is that proteolytic processing "enhances" the fusion activity of the MHV S protein. The selective advantage of an enhanced fusogenic potential may be manifest in the extensive development of syncytia in vivo and the spread of virus from cell to cell without exposure to the humoral immune response. It is possible however that cleavage or the extent of cleavage is also an essential factor governing tropism and pathogenesis. For example the $S$ protein of the neurotropic Coronavirus MHV-4 shows a different cleavage pattern compared to the $S$ protein of MHV-JHM (21).

In our opinion, however, answers to questions such as these require the development of an infectious MHV cDNA clone or a system of targeted recombination (22). With such a system, it would be possible to introduce specific changes into the MHV genome and analyse their effect on the virus phenotype in vitro and in vivo.

\section{REFERENCES}

1. Spaan, W.J.M., Cavanagh, D. \& Horzinek, M.C. (1988). Journal of General Virology 69, 2939 2952.

2. Lee, H.-J., Shieh, C.-K., Gorbalenya, A.E., Koonin, E.V., LaMonica, N., Tuler, J., Bagdzhadzhyan, A. \& Lai, M.M.C. (1991). Virology 180, 567-582.

3. Siddell, S.G. (1982). Journal of General Virology 62, 259-269.

4. Schmidt, I., Skinner, M. \& Siddell, S. (1987). Journal of General Virology 68, 47-56.

5. Collins, A.R., Knobler, R.L., Powell, H. \& Buchmeier, M.J. (1982). Virology 119, 358-371.

6. Bosch, F.X., Orlich, M., Klenk, H.-D. \& Rott, R. (1979). Virology 95, 197-207.

7. Bosch, V.,\& Rawlita, M. (1990). Journal of Virology 64, 2337-2344.

8. Yoshikura, H. \& Tejima, S. (1981). Virology 113, 503-511.

9. Sturman, L.S., Ricard, C.S. \& Holmes, K.V. (1985). Journal of Virology. 56, 904-911.

10. Frana, M.F., Behnke, J.N., Sturman, L.S. \& Holmes, K.V. (1985). Journal of Virology 56, 912 920.

11. de Groot, R.J., van Leen, R.W., Dalderup, M.J.M., Vennema, H., Horzinek, M.C. \& Spaan, W.J.M. (1989). Virology, 171 493-502.

12. Schwarz, B., Routledge, E. \& Siddell, S.G. (1990). Journal of Virology 64, 4784-4791.

13. Fuerst, T.R., Niles, E.G., Studier, F.W. \& Moss, B. (1986). Proceedings of the National Academy of Sciences, USA 83, 8122-8126.

14. Mackett, M., Smith, G.L. \& Moss, B. (1985). In "DNA Cloning: A Practical Approach", pp191211. Edited by D.M. Glover, Oxford, IRL Press.

15. Gubler, U. \& Hoffman, B.J. (1983). Gene 25, 263-269.

16. Fuerst, T.R., Earl, P.L. \& Moss, B. (1987). Molecular and Cellular Biology 7, 2538-2544.

17. Routledge, E., Stauber, R., Pfleiderer, M. \& Siddell, S.G. (1991). Journal of Virology 65, 254 262.

18. Wege, H., Dorries, R. \& Wege, H. (1984). Journal of General Virology 65, 1931-1942.

19. Kooi, C., Cervin, M. \& Anderson, R. (1991). Virology 180, 108-119.

20. Mizzen, L. Cheley, S., Rao, M., Wolf, R. \& Anderson, R. (1983). Virology 128, 407-417.

21. Gallagher, T.M., Parker, S.E. \& Buchmeier, M.J. (1990). Journal of Virology 64, 731-744.

22. Koetzner, C.A., Parker, M.M., Ricard, C.S., Sturman, L.S. \& Masters, P.S. (1992). Journal of Virology $66,1841-1848$. 